\title{
Investigation of Prefrontal Cortex Activity in University Students with Presenteeism: A Near-Infrared Spectroscopy (NIRS) Study
}

\author{
Masateru Matsushita1 ${ }^{1 *}$, Schuhei Yamamura ${ }^{2,3}$, Manabu Ikeda ${ }^{4}$ \\ ${ }^{1}$ Center for Medical Education and Research, Faculty of Life Sciences, Kumamoto University, Kumamoto, Japan \\ ${ }^{2}$ Department of Psychiatry, Kinki Central Hospital, Itami, Japan \\ ${ }^{3}$ Osaka University Health Care Center, Toyonaka, Japan \\ ${ }^{4}$ Department of Neuropsychiatry, Faculty of Life Sciences, Kumamoto University, Kumamoto, Japan \\ Email: ${ }^{*}$ matsushita@fc.kuh.kumamoto-u.ac.jp
}

Received 18 July 2015; accepted 15 August 2015; published 18 August 2015

Copyright (C) 2015 by authors and Scientific Research Publishing Inc.

This work is licensed under the Creative Commons Attribution-NonCommercial International License (CC

BY-NC).

http://creativecommons.org/licenses/by-nc/4.0/

(c) () () O) Open Access

\begin{abstract}
Presenteeism refers to impaired performance attributed to attending work with health problems. There has been no study examining the state of presenteeism with objective measures. We compared cerebral hemodynamic changes, measured by near-infrared spectroscopy (NIRS), during neuropsychological tests conducted by university students with presenteeism and healthy controls. Twenty-two university students participated in the study; 11 of them with impaired performance caused by mental health problem were allocated to the presenteeism group and 11 without health problems to the control group. Presenteeism was assessed by the Presenteeism Scale for Students. To evoke hemodynamics changes, the participants completed a Word Fluency Test (WFT) and a Trail Making Test (TMT). The NIRS probes were located over the bilateral prefrontal area. Students with presenteeism had significantly higher incidences of depression than controls. However, there was no significant difference in behavioral performance examinations between the two groups. With regard to hemodynamics changes, the repeated measures analysis of covariance of the NIRS signals revealed significant interactions between group and task activation. Although we observed a significant increase in oxygenated hemoglobin concentration during the WFT among controls (simple main effect; left channel, $F_{(1,19)}=27.34, P<0.001$; right channel, $F_{(1,19)}=22.05, P<$ 0.001), no changes were found in students with presenteeism during either the WFT (simple main effect; left channel, $F_{(1,19)}=0.12, P<0.732$; right channel, $F_{(1,19)}=0.08, P<0.778$ ) or TMT tasks (left channel, $t=-0.94, P$ with Bonferroni correction $=0.745$; right channel, $t=-2.19, P$ with Bonferroni correction $<0.113$ ). This is the first study to reveal differences in activity in the cerebral cortex associated with presenteeism. The fact that students with presenteeism have prefrontal dysfunction might reinforce the concept of presenteeism.
\end{abstract}

${ }^{*}$ Corresponding author.

How to cite this paper: Matsushita, M., Yamamura, S. and Ikeda, M. (2015) Investigation of Prefrontal Cortex Activity in University Students with Presenteeism: A Near-Infrared Spectroscopy (NIRS) Study. Journal of Behavioral and Brain Science, 5, 339-347. http://dx.doi.org/10.4236/jbbs.2015.59034 
Keywords

Absenteeism, Adolescent Health, Near-Infrared Spectroscopy (NIRS), Presenteeism, School Refusal

\section{Introduction}

Presenteeism is the loss of performance in the work place due to health problems [1] [2]. During the last two decades, a number of studies concerning presenteeism have been conducted in the field of occupational health [3]-[5]. Recently, Bergström and colleagues (2009) reported that presenteeism adversely affected general health three years later [6]. Presenteeism is also related to future absenteeism due to sickness [7]. The state of presenteeism may thus be an important factor contributing to a long-term sickness, absenteeism, or job resignation.

It is thought that the proportion of university students who attend class even though they have identified health problems is not negligible [8]. In fact, we found that approximately $60 \%$ of university students had experienced some health problems that contributed to weakened academic performance, and proposed this condition as "presenteeism in students" [9] [10]. Good health care in adolescence might be crucial to decreasing the risk of absenteeism and further morbidity because many lifestyle-related health problems are formed at this phase and persist later in life [11]. Hence, more attention should be given to the issue of presenteeism in university students.

To our knowledge, all previous studies regarding presenteeism were based on self-administered questionnaires. No studies examined whether there were any physiological differences in cerebral activity in either students or workers. To develop a deeper understanding of the concept of presenteeism, it is important to examine possible physiological changes associated with the condition.

Of the commonly used functional neuroimaging methods, including single photon emission computed tomography (SPECT), positron emission tomography (PET), functional magnetic resonance imaging (fMRI), and near-infrared spectroscopy (NIRS), NIRS is especially useful for assessing functional changes in the cortex by measuring hemodynamic responses, and is favorable to psychiatric patients because it is quite small, non-invasive, and easy to operate. Attenuated responses in oxygenated hemoglobin (oxy-Hb) during cognitive stimulations have been observed in psychiatric patients, including those with schizophrenia and depression [12]-[14].

The purpose of this study was, therefore, to assess the state of presenteeism using objective and biological measuring methods. We investigated changes in cerebral hemodynamics during neuropsychological tests conducted by university students with presenteeism and healthy controls.

\section{Methods}

\subsection{Participants and Procedure}

Eight university students who visited the health care center for a consultation about mental health problems were enrolled in the study, and an additional 14 student volunteers were enrolled through a lecture about mental health. Of these 22 participants, 11 who had some kind of psychological problems such as depression, anxiety or emotional disorder were evaluated by the Presenteeism Scale for Students (PSS) and allocated to the "Presenteeism group" [9]. The other 11 students had no health problems and were designated as the "Control group". The experimental study concerning the association of presenteeism with cognitive function was conducted from June 2012 to October 2012.

Participants were asked to maintain a routine for bedtime and rising and not to drink alcoholic beverages on the night before the experiment. After administering the PSS, Beck Depression Inventory second edition (BDIII), and NEO Five-Factor Inventory (NEO-FFI) questionnaires on the experimental day, we assessed executive function via the verbal Word Fluency Test (WFT) and Trail Making Test (TMT Part A and B). After providing a written explanation of the aim of the study, informed consent was obtained from all participants. The study protocol was approved by the Ethics Committee of the Osaka University Health Care Center.

\subsection{Questionnaires}

Firstly, the PSS was used to assess the health problems of participants and the severity of presenteeism. The in- 
ternal consistency, test-retest reliability, factorial validity, and concurrent validity of PSS have been well addressed previously [9].

Secondly, depression was assessed using the Japanese version of BDI-II, which consists of 21 items [15] [16]. Scores for this instrument rage from 0 to 63, with higher scores indicating greater depressive symptoms; the Japanese version of BDI-II was reported to have good reliability and validity [16].

Finally, personality was assessed using the Japanese version of NEO-FFI, a 60-item scale designed to assess the following five personality traits: neuroticism, extraversion, openness to experience, agreeableness, and conscientiousness. Each dimensional score is presented as a T-score with a mean of 50 and a standard deviation of 10. This scale has also been shown to have adequate reliability, internal consistency, and validity [17].

\subsection{NIRS Measurement}

NIRS easily and non-invasively measures brain activity by monitoring changes in hemodynamic responses when neurons are activated. In the area of activated brain neurons, oxygen consumption gradually increases and blood supply increases to provide oxygen via oxygenated hemoglobin (oxy-Hb). NIRS takes advantage of the fact that blood hemoglobin diffuses near-infrared light, and, furthermore, that the absorption and diffusion of near-infrared light depend on the degree of oxidation of the hemoglobin. By measuring the transmitted near-infrared light between emitter and receiver probes, we can estimate the change in brain metabolic activity. In this study, metabolic neural activity was assessed by the oxy-Hb, deoxygenated hemoglobin (deoxy- $\mathrm{Hb}$ ), and total $\mathrm{Hb}$, all indicators of changes in blood within the irradiated area.

We used a 2-channel miniaturized wireless instrument (PocketNIRS Duo, DynaSense, Japan), which utilizes near-infrared light emitted at three different wavelengths $(735,810$, and $850 \mathrm{~nm})$ based on the modified BeerLambert law. We measured activity within the prefrontal cortex through 2 pairs of light source and detection probes located at Fp1 and Fp2 according to the international 10 - 20 electrode placement system for electroencephalography of Rechtschaffen and Kales [18]. The distance between the light source and detection probes was $3 \mathrm{~cm}$. Sampling frequency of the absorption of near-infrared light was set as $10 \mathrm{~Hz}$ (a time resolution of $0.1 \mathrm{~s}$ ). The data from NIRS is expressed in arbitrary units.

\subsection{Neuropsychological Task}

Brain activation was evoked by the verbal WFT and TMT tasks [19] [20]. Participants sat on a comfortable chair in a silent room. In the verbal WFT task, the participants were asked to name (within a 60-s period per letter) as many nouns as they could beginning with the Japanese hiragana letters "fu", "a", and "ni". The sequence of the hiragana letters was counterbalanced among 3 groups of randomly allocated participants. The total number of words produced during the task periods was taken as behavioral performance. Because NIRS data vary greatly from individual to individual, an intra-individual comparison is preferable to a between-groups comparison. We, therefore, also collected NIRS data during a baseline period (see Figure 1), during which the participants were instructed to vocalize the letters "a", "i”, “u”, "e”, "o", repeatedly for 30 s. An auditory cue was provided to signal the switch to each given task period from the baseline period. To prevent postural changes from evoking movement-related artifacts in the NIRS data, participants were instructed to focus on a black point in front of their desk during both the baseline and WFT periods.

The TMT task, consisting of Parts A and B, is a widely used task for evaluating executive function [21]. In the Part A, the participants are required to draw a line connecting consecutive numbers $(1-25)$ in sequential order as rapidly as possible. In Part $\mathrm{B}$, they must draw a line alternately between consecutive numbers and letters (1, A, 2, B, 3, etc.) as quickly as possible. As a control task, the participants must draw a line connecting sequentially presented numbers. The time required to complete the task was the measure of behavioral performance.

\subsection{Data Analysis}

For the WFT, we analyzed the last $20 \mathrm{~s}$ of the NIRS baseline period prior to the start period for each letter of the task, calculated the average changes for these three baseline periods, and defined this as the baseline data. For the activation periods, we analyzed the last $50 \mathrm{~s}$ of the WFT task period. For baseline-correction of the NIRS data, the mean values of oxy-Hb, deoxy-Hb, and total $\mathrm{Hb}$ were set to zero for each block (see Figure 1). The 


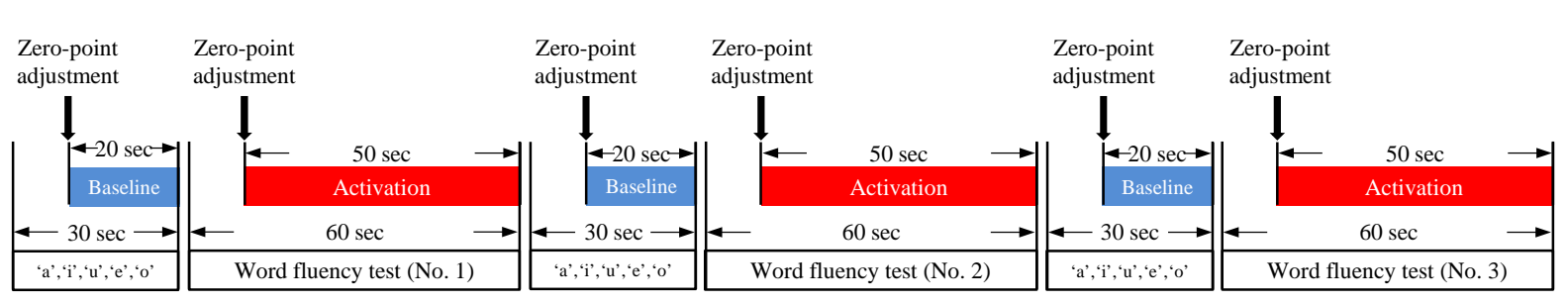

(a) Time course of the word fluency test

Red blocks represent activation periods.

Blue blocks represent the baseline periods for NIRS data analysis.

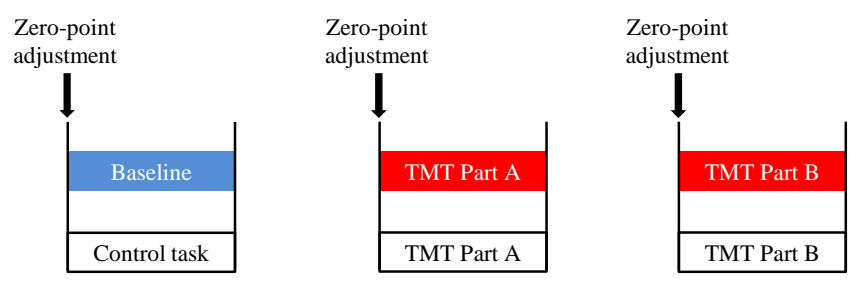

(b) Time course of the trail making tests

Blue and red blocks indicate the periods during which NIRS data was analyzed.

The durations of the baseline and task periods varied by individual.

Figure 1. Time courses and data analyses of the word fluency test and trail making test.

averaged changes for the three periods during which the participants were engaged in the WFT was used as the activation data. The baseline and activation data for the levels of oxy-Hb, deoxy- $\mathrm{Hb}$, and total $\mathrm{Hb}$ were then used in statistical analyses.

For the TMT task, we included all epochs of the control task, TMT Part A, and Part B in statistical analyses, because the length of each part was different. Average values during the control task, TMT Part A, and Part B were calculated and used for statistical analyses (see Figure 1).

The chi-square test and $t$-test were used for group comparisons. For analysis of relationships between presenteeism and brain activity, we calculated the Spearman's rank correlation coefficient. We also performed a repeated measures analysis of covariance (ANCOVA), with age as a covariate, to compare the hemodynamic changes between baseline and activation condition. If ANCOVA indicated a significant interaction, we conducted a simple main effect. We performed paired $t$-tests to assess hemodynamic changes during the TMT task. The $P$ values of the pairwise comparisons were corrected by Bonferroni method. All statistical analyses were executed using IBM SPSS Statistics 20.0.0.1 statistical software. We considered a 2-tailed $P$ value of less than 0.05 to indicate a statistically significant difference.

\section{Results}

Comparisons of demographics, psychological variables, and behavioral performances on the neuropsychological tests between controls and presenteeism students are summarized in Table 1. Although significant differences in age ( $t=-2.86, P=0.014)$ and BDI score $(t=-2.48, P=0.027)$ were found, there were no significant differences in personality traits or behavioral performances on the WFT and TMT tasks. Work impairment scores (WIS) on the PSS, which reflect the impact of presenteeism on performance, were significantly correlated with BDI scores in the presenteeism group $(\rho=0.88, P<0.001)$ (Figure 2). Students in the presenteeism group with lower WIS scores exhibited higher extraversion $(\rho=-0.68, P=0.023)$ and agreeableness $(\rho=-0.66, P=0.028)$ on the NEO-FFI (Figure 2).

Table 2 summarizes the changes in oxy- $\mathrm{Hb}$, deoxy- $\mathrm{Hb}$, and total $\mathrm{Hb}$ between the baseline and activation conditions of the WFT task. The repeated measures ANCOVA of oxy-Hb revealed significant interactions between group and task activation; the simple main effect of task activation was significant in the healthy controls but not in the presenteeism group. Performing the WFT induced a significant increase in oxy-Hb only in the control group. There were also significant main effects of group in deoxy-Hb. Students with presenteeism had higher 


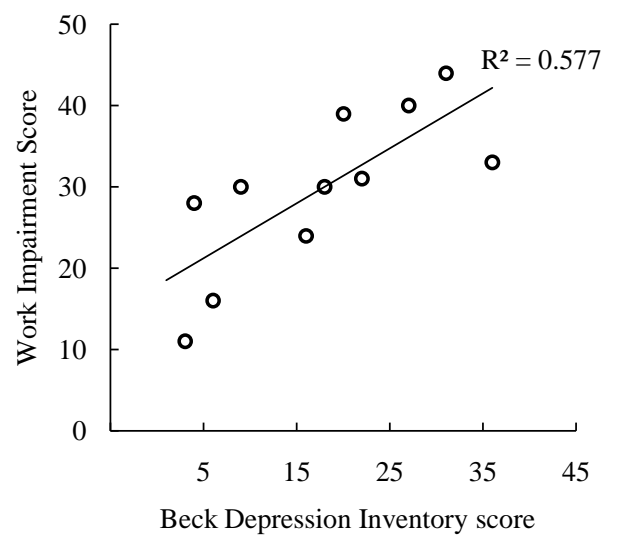

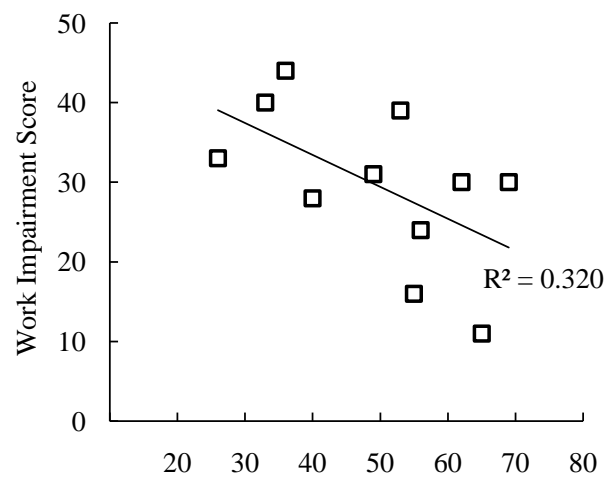

Personality trait-Agreeableness

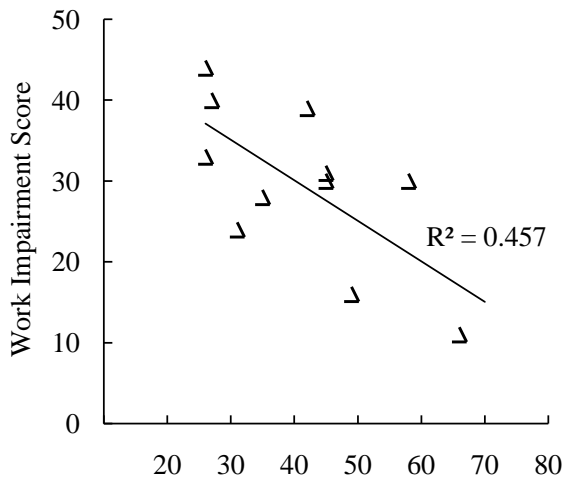

Personality trait-Extraversion

Figure 2. Scatter plots for correlations of the degree of presenteeism to depression and personality traits.

Table 1. Characteristics of the study groups.

\begin{tabular}{|c|c|c|c|c|c|c|}
\hline & \multicolumn{2}{|c|}{ Controls } & \multicolumn{2}{|c|}{ Presenteeism } & \multirow{2}{*}{$t / \chi^{2}$} & \multirow{2}{*}{$P$} \\
\hline & Mean & SD & Mean & SD & & \\
\hline Age & 19.8 & 1.17 & 22.7 & 3.20 & 2.84 & $0.014^{*}$ \\
\hline Sex (percentage of male) & \multicolumn{2}{|c|}{$36.4 \%$} & \multicolumn{2}{|c|}{$54.5 \%$} & 0.73 & 0.670 \\
\hline BDI score & 8.4 & 4.91 & 17.5 & 11.14 & 2.48 & $0.027^{*}$ \\
\hline \multicolumn{7}{|l|}{ NEO-FFI } \\
\hline Neuroticism & 43.8 & 10.07 & 49.5 & 10.46 & 1.29 & 0.213 \\
\hline Extraversion & 49.8 & 11.56 & 40.9 & 13.39 & 1.67 & 0.110 \\
\hline Openness to experience & 51.3 & 5.98 & 51.1 & 8.98 & 0.06 & 0.956 \\
\hline Agreeableness & 46.0 & 11.39 & 49.5 & 14.00 & 0.64 & 0.533 \\
\hline Conscientiousness & 49.5 & 9.68 & 50.2 & 6.68 & 0.21 & 0.840 \\
\hline \multicolumn{7}{|l|}{ Word fluency test } \\
\hline Task performance & 28.0 & 11.84 & 26.5 & 5.54 & 0.39 & 0.701 \\
\hline \multicolumn{7}{|l|}{ Trail making test } \\
\hline Part A (s) & 29.3 & 11.13 & 28.4 & 10.91 & 0.18 & 0.846 \\
\hline Part B (s) & 54.7 & 16.22 & 52.0 & 14.72 & 0.41 & 0.683 \\
\hline
\end{tabular}

${ }^{*} P<0.05$. The chi-square test or $t$ test were used for group comparisons. 
Table 2. Hemodynamic changes induced by word fluency test in students with presenteeism and healthy controls.

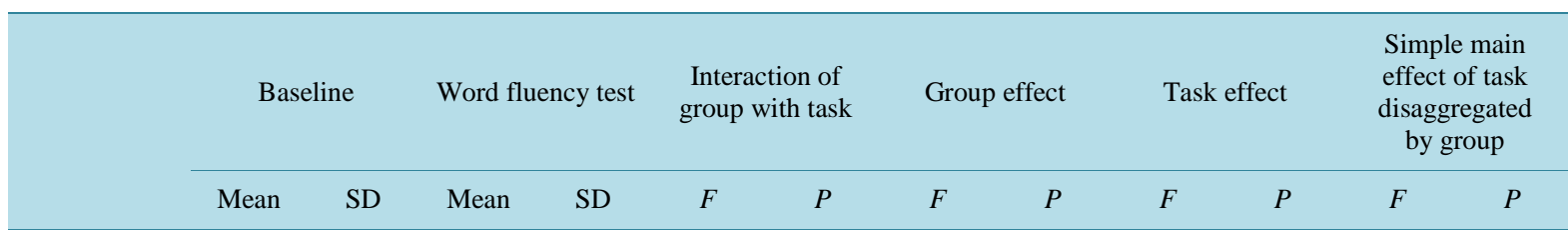

$\mathrm{Oxy}-\mathrm{Hb}$

Left channel

\begin{tabular}{|c|c|c|c|c|c|c|c|c|c|c|c|c|}
\hline Controls & -0.0019 & $(0.005)$ & 0.0337 & $(0.020)$ & 13.32 & $0.002^{* *}$ & 7.73 & $0.012^{*}$ & 0.22 & 0.645 & 27.34 & $<0.001^{* * *}$ \\
\hline Presenteeism & -0.0004 & $(0.007)$ & -0.0003 & $(0.022)$ & & & & & & & 0.12 & 0.732 \\
\hline \multicolumn{13}{|l|}{ Right channel } \\
\hline Controls & -0.0022 & $(0.006)$ & 0.0272 & $(0.017)$ & 10.63 & $0.004^{* *}$ & 7.86 & $0.011^{*}$ & 0.42 & 0.524 & 22.05 & $<0.001^{* * *}$ \\
\hline Presenteeism & -0.0016 & $(0.004)$ & -0.0007 & $(0.021)$ & & & & & & & 0.08 & 0.778 \\
\hline
\end{tabular}

Deoxy-Hb

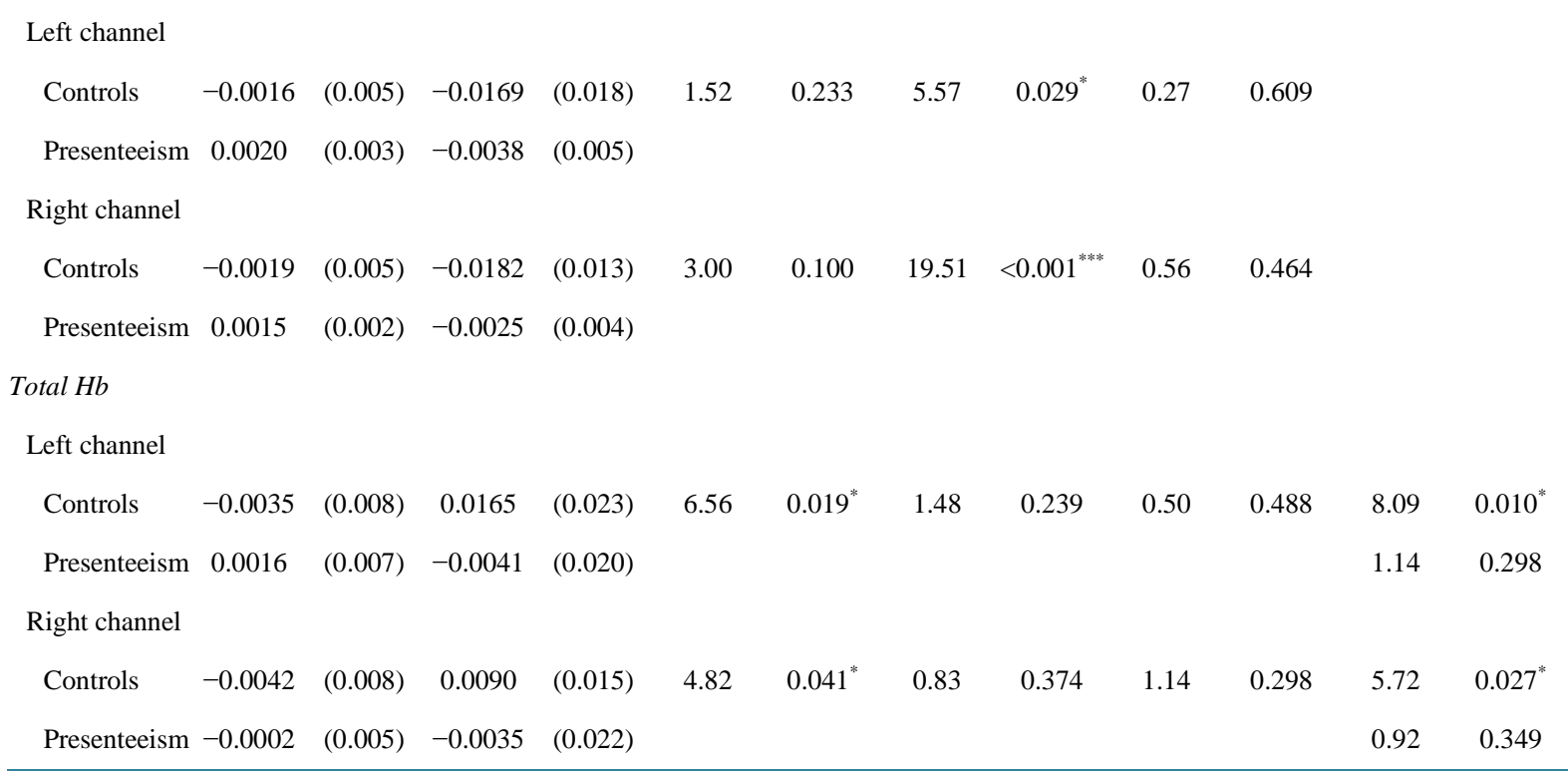

${ }^{* * *} P<0.001,{ }^{* *} P<0.01,{ }^{*} P<0.05$ significant differences between control and presenteeism groups (ANCOVA followed by test for simple main effec). Degrees of freedom for $F$-value were $F(1,19)$ for all reported effects.

deoxy-Hb compared to healthy controls. A significant interaction of group with task activation was also found for the changes in total $\mathrm{Hb}$; there was a significant increase in total $\mathrm{Hb}$ only in controls.

Table 3 summarizes the hemodynamic changes during the TMT task. We found the significant increasing during TMT Part B in the healthy control students.

\section{Discussion}

We examined whether it was possible to associate brain activity with presenteeism, which is a subjective symptom, by means of objective measurements. Specifically, we used NIRS as a functional neuroimaging method to examine activity in the prefrontal cortex induced by cognitive stimuli in presenteeism students and healthy controls. The students with presenteeism did not show significant increases in prefrontal cortex activation during the WFT and TMT tasks, whereas healthy controls did. Previous studies reported that healthy controls had a greater increase in oxy-Hb under various cognitive stimulations in prefrontal cortex compared to patients with depression [22] [23], schizophrenia [24], and pervasive developmental disorder [25] [26]. Our results are quite similar 
Table 3. Hemodynamic changes induced by the trail making test in students with presenteeism and healthy controls.

\begin{tabular}{|c|c|c|c|c|c|c|c|c|}
\hline & \multicolumn{2}{|c|}{ TMT control } & \multicolumn{2}{|c|}{ TMT Part A } & \multicolumn{2}{|c|}{ TMT Part B } & \multirow{2}{*}{$\begin{array}{l}P \text { for control } \\
\text { vs Part A }\end{array}$} & \multirow{2}{*}{$\begin{array}{l}P \text { for control } \\
\text { vs Part B }\end{array}$} \\
\hline & Mean & SD & Mean & SD & Mean & SD & & \\
\hline \multicolumn{9}{|l|}{$\mathrm{Oxy}-\mathrm{Hb}$} \\
\hline \multicolumn{9}{|l|}{ Controls } \\
\hline Left channel & 0.0040 & $(0.009)$ & 0.0464 & $(0.091)$ & 0.0299 & $(0.033)$ & 0.243 & $0.021^{*}$ \\
\hline Right channel & 0.0057 & $(0.005)$ & 0.0415 & $(0.079)$ & 0.0298 & $(0.027)$ & 0.323 & $0.032^{*}$ \\
\hline \multicolumn{9}{|l|}{ Presenteeism } \\
\hline Left channel & -0.0029 & $(0.008)$ & 0.0031 & $(0.008)$ & 0.0007 & $(0.011)$ & 0.433 & 0.745 \\
\hline Right channel & -0.0033 & $(0.008)$ & 0.0047 & $(0.014)$ & 0.0050 & $(0.006)$ & 0.421 & 0.113 \\
\hline \multicolumn{9}{|l|}{ Deoxy-Hb } \\
\hline \multicolumn{9}{|l|}{ Controls } \\
\hline Left channel & -0.0001 & $(0.006)$ & -0.0048 & $(0.020)$ & -0.0011 & $(0.012)$ & 1.079 & 1.662 \\
\hline Right channel & -0.0018 & $(0.006)$ & -0.0116 & $(0.026)$ & -0.0139 & $(0.015)$ & 0.616 & 0.107 \\
\hline \multicolumn{9}{|l|}{ Presenteeism } \\
\hline Left channel & 0.0046 & $(0.011)$ & -0.0043 & $(0.009)$ & -0.0066 & $(0.012)$ & 0.082 & 0.126 \\
\hline Right channel & 0.0006 & $(0.008)$ & -0.0024 & $(0.003)$ & -0.0055 & $(0.007)$ & 0.679 & 0.142 \\
\hline \multicolumn{9}{|l|}{ Total $\mathrm{Hb}$} \\
\hline \multicolumn{9}{|l|}{ Controls } \\
\hline Left channel & 0.0038 & $(0.013)$ & 0.0415 & $(0.078)$ & 0.0288 & $(0.039)$ & 0.201 & 0.076 \\
\hline Right channel & 0.0040 & $(0.007)$ & 0.0297 & $(0.055)$ & 0.0158 & $(0.017)$ & 0.233 & 0.055 \\
\hline \multicolumn{9}{|l|}{ Presenteeism } \\
\hline Left channel & 0.0020 & $(0.014)$ & -0.0027 & $(0.014)$ & -0.0060 & (0.019) & 0.949 & 0.768 \\
\hline Right channel & -0.0026 & $(0.009)$ & 0.0025 & $(0.013)$ & -0.0005 & $(0.005)$ & 0.557 & 0.948 \\
\hline
\end{tabular}

${ }^{*} P<0.05$. All $P$ values were adjusted by Bonferroni correction.

to these previous studies. These previous findings may help to explain why the students with presenteeism did not exhibit increased prefrontal cortex activity during cognitive tasks.

In the present study, although we did not find significant differences in behavioral performances on the WFT and TMT tasks between students with presenteeism and controls, increased activation of the prefrontal cortex during these tasks was identified by NIRS only in healthy students. It is thought that we objectively disclosed a loss of productivity that is observed in presenteeism using a neuroimaging method. Another significant point is that the BDI scores and personality traits were significantly associated with WIS, which represents the degree of presenteeism. In particular, extraversion and agreeableness, which were inversely correlated with WIS, may be important factors influencing the prevention and remedy of presenteeism. Furthermore, these correlation-based results might reinforce the concurrent validation of the PSS.

Moreover, we consider that the present study is particularly informative in representing the concept of presenteeism. In our previous study, we confirmed the reliability of the PSS via the test-retest method as well as its concurrent and factorial validity [9]. In this study, we have verified the construct validity of the PSS via the NIRS measurements, and demonstrated that students with presenteeism exhibited a dysfunction in the prefrontal cortex.

Despite the lack of decreased behavioral performance, the fact that cortical brain dysfunction underlying the performance might occur, seems to be important for university students because high performance is required to 
conduct university-level study. Another study using NIRS showed that memory learning increased oxy-Hb concentrations in healthy participants, and suggested that the activation of prefrontal cortex during memory learning plays a critical role in verbal memory or learning [27]. In other words, the students with presenteeism exhibited less activation in the prefrontal cortex even when they required attentional concentration, which might result in low productivity. Furthermore, presenteeism might lead to a lower quality of campus life, accidents resulting from inattention during experiments, or dropping out of the university.

\section{Conclusion}

In conclusion, this study is the first to reveal an association of presenteeism with activity of the cerebral cortex. We believe that the fact, students with presenteeism have prefrontal dysfunction, might reinforce the concept of presenteeism.

\section{Acknowledgements}

This study was supported by a Grant-in-Aid for Young Scientists (B) (No. 23700799 to SY) from the Ministry of Education, Science, Sports and Culture of Japan. We would like to express our sincere gratitude to Nakamori Suganuma, Ph.D. (Osaka University Health Care Center) for conducting the surveys.

\section{Conflicts of Interest}

The authors report no conflicts of interest.

\section{References}

[1] Yamashita, M. and Arakida, M. (2006) Concept Analysis of Presenteeism and Its Possible Applications in Japanese Occupational Health. Sangyo Eiseigaku Zasshi, 48, 201-213. (In Japanese) http://dx.doi.org/10.1539/sangyoeisei.48.201

[2] Auren, U. (1955) How to Build Presenteeism. Petroleum Refiner, 34, 348-359.

[3] Koopman, C., Pelletier, K.R., Murray, J.F., Sharda, C.E., Berger, M.L., Turpin, R.S., Hackleman, P., Gibson, P., Holmes, D.M. and Bendel, T. (2002) Stanford Presenteeism Scale: Health Status and Employee Productivity. Journal of Occupational and Environmental Medicine, 44, 14-20. http://dx.doi.org/10.1097/00043764-200201000-00004

[4] Goetzel, R.Z., Long, S.R., Ozminkowski, R.J., Hawkins, K., Wang, S. and Lynch, W. (2004) Health, Absence, Disability, and Presenteeism Cost Estimates of Certain Physical and Mental Health Conditions Affecting U.S. Employers. Journal of Occupational and Environmental Medicine, 46, 398-412. http://dx.doi.org/10.1097/01.jom.0000121151.40413.bd

[5] Wada, K., Arakida, M., Watanabe, R., Negishi, M., Sato, J. and Tsutsumi, A. (2013) The Economic Impact of Loss of Performance Due to Absenteeism and Presenteeism Caused by Depressive Symptoms and Comorbid Health Conditions among Japanese Workers. Industrial Health, 51, 482-489. http://dx.doi.org/10.2486/indhealth.2013-0016

[6] Bergström, G., Bodin, L., Hagberg, J., Lindh, T., Aronsson, G. and Josephson, M. (2009) Does Sickness Presenteeism Have an Impact on Future General Health? International Archives of Occupational and Environmental Health, 82, 1179-1190. http://dx.doi.org/10.1007/s00420-009-0433-6

[7] Janssens, H., Clays, E., Clercq, B.D., Bacquer, D.D. and Braeckman, L. (2013) The Relation between Presenteeism and Different Types of Future Sickness Absence. Journal of Occupational Health, 55, 132-141. http://dx.doi.org/10.1539/joh.12-0164-OA

[8] Hussain, R., Guppy, M., Robertson, S. and Temple, E. (2013) Physical and Mental Health Perspectives of First Year Undergraduate Rural University Students. BMC Public Health, 13, 848-859. http://dx.doi.org/10.1186/1471-2458-13-848

[9] Matsushita, M., Adachi, H., Arakida, M., Namura, I., Takahashi, Y., Miyata, M., Kumano-go, T., Yamamura, S., Shigedo, Y., Suganuma, N., Mikami, A., Moriyama, T. and Sugita, Y. (2011) Presenteeism in College Students: Reliability and Validity of the Presenteeism Scale for Students. Quality of Life Research, 20, 439-446. http://dx.doi.org/10.1007/s11136-010-9763-9

[10] Mikami, A., Matsushita, M., Adachi, H., Suganuma, N., Koyama, A., Ichimi, N., Ushijima, H., Ikeda, M., Takeda, M., Moriyama, T. and Sugita, Y. (2013) Sense of Coherence, Health Problems, and Presenteeism in Japanese University Students. Asian Journal of Psychiatry, 6, 369-372. http://dx.doi.org/10.1016/j.ajp.2013.03.008

[11] Sawyer, S.M., Afifi, R.A., Bearinger, L.H., Blakemore, S.J., Dick, B., Ezeh, A.C. and Patton, G.C. (2012) Adolescence: 
A Foundation for Future Health. The Lancet, 28, 1630-1640. http://dx.doi.org/10.1016/S0140-6736(12)60072-5

[12] Azechi, M., Iwase, M., Ikezawa, K., Takahashi, H., Canuet, L., Kurimoto, R., Nakahachi, T., Ishii, R., Fukumoto, M., Ohi, K., Yasuda, Y., Kazui, H., Hashimoto, R. and Takeda, M. (2010) Discriminant Analysis in Schizophrenia and Healthy Subjects Using Prefrontal Activation during Frontal Lobe Tasks: A Near-Infrared Spectroscopy. Schizophrenia Research, 117, 52-60. http://dx.doi.org/10.1016/j.schres.2009.10.003

[13] Suto, T., Fukuda, M., Ito, M., Uehara, T. and Mikuni, M. (2004) Multichannel Near-Infrared Spectroscopy in Depression and Schizophrenia: Cognitive Brain Activation Study. Biological Psychiatry, 55, 501-511. http://dx.doi.org/10.1016/j.biopsych.2003.09.008

[14] Kameyama, M., Fukuda, M., Yamagishi, Y., Sato, T., Uehara, T., Ito, M., Suto, T. and Mikuni, M. (2006) Frontal Lobe Function in Bipolar Disorder: A Multichannel Near-Infrared Spectroscopy Study. Neuroimage, 29, 172-184. http://dx.doi.org/10.1016/j.neuroimage.2005.07.025

[15] Beck, A.T., Steer, R.A. and Brown, G.K. (1996) BDI-II Manual. The Psychological Corporation, San Antonio.

[16] Kojima, M., Furukawa, T.A., Takahashi, H., Kawai, M., Nagaya, T. and Tokudome, S. (2002) Cross-Cultural Validation of the Beck Depression Inventory-II in Japan. Psychiatry Res, 110, 291-299. http://dx.doi.org/10.1016/S0165-1781(02)00106-3

[17] Costa, P.T. and McCrae, R.R. (1992) Revised NEO Personality Inventory (NEO-PI-R) and NEO Five-Factor Inventory (NEO-FFI) Professional Manual. Psychological Assessment Resources, Odessa, FL.

[18] Rechtschaffen, A. and Kales, A. (1968) A Manual of Standardized Terminology, Techniques and Scoring System of Sleep Stages in Human Subjects. University of California, Los Angeles.

[19] Lezak, M.D. (1995) Neuropsychological Assessment. Fifth Edition, Oxford University Press, New York, $422-425$.

[20] Lezak, M.D. (1995) Neuropsychological Assessment. Fifth Edition, Oxford University Press, New York, 693-694.

[21] Shibuya-Tayoshi, S., Sumitani, S., Kikuchi, K., Tanaka, T., Tayoshi, S., Ueno, S. and Ohmori, T. (2007) Activation of the Prefrontal Cortex during the Trail-Making Test Detected with Multichannel Near-Infrared Spectroscopy. Psychiatry and Clinical Neurosciences, 61, 616-621. http://dx.doi.org/10.1111/j.1440-1819.2007.01727.x

[22] Ikeda, E., Shiozaki, K., Ikeda, H., Suzuki, M. and Hirayasu, Y. (2013) Prefrontal Dysfunction in Remitted Depression at Work Reinstatement Using Near-Infrared Spectroscopy. Psychiatry Research: Neuroimaging, 214, 254-259. http://dx.doi.org/10.1016/j.pscychresns.2013.07.009

[23] Pu, S., Matsumura, H., Yamada, T., Ikezawa, S., Mitani, H., Adachi, A. and Nakagome, K. (2008) Reduced Frontopolar Activation during Verbal Fluency Task Associated with Poor Social Functioning in Late-Onset Major Depression: Multi-Channel Near-Infrared Spectroscopy Study. Psychiatry and Clinical Neurosciences, 62, 728-737. http://dx.doi.org/10.1111/j.1440-1819.2008.01882.x

[24] Ikezawa, K., Iwase, M., Ishii, R., Azechi, M., Canuet, L., Ohi, K., Yasuda, Y., Iike, N., Kurimoto, R., Takahashi, H., Nakahachi, T., Sekiyama, R., Yoshida, T., Kazui, H., Hashimoto, R. and Takeda, M. (2009) Impaired Regional Hemodynamic Response in Schizophrenia during Multiple Prefrontal Activation Tasks: A Two-Channel Near-Infrared Spectroscopy Study. Schizophrenia Research, 108, 93-103. http://dx.doi.org/10.1016/j.schres.2008.12.010

[25] Iwanaga, R., Tanaka, G., Nakane, H., Honda, S., Imamura, A. and Ozawa, H. (2013) Usefulness of Near-Infrared Spectroscopy to Detect Brain Dysfunction in Children with Autism Spectrum Disorder When Inferring the Mental State of Others. Psychiatry and Clinical Neurosciences, 67, 203-209. http://dx.doi.org/10.1111/pcn.12052

[26] Nakadoi, Y., Sumitani, S., Watanabe, Y., Akiyama, M., Yamashita, N. and Ohmori, T. (2012) Multi-Channel NearInfrared Spectroscopy Shows Reduced Activation in the Prefrontal Cortex during Facial Expression Processing in Pervasive Developmental Disorder. Psychiatry and Clinical Neurosciences, 66, 26-33. http://dx.doi.org/10.1111/j.1440-1819.2011.02290.x

[27] Matsui, M., Tanaka, K., Yonezawa, M. and Kurachi, M. (2007) Activation of the Prefrontal Cortex during Memory Learning: Near-Infrared Spectroscopy Study. Psychiatry and Clinical Neurosciences, 61, 31-38. http://dx.doi.org/10.1111/j.1440-1819.2007.01607.x 